\title{
Surface States and Spectra
}

\author{
Vojkan Jakšićc ${ }^{1 *}$ and Yoram Last ${ }^{2,3}$ \\ ${ }^{1}$ Department of Mathematics \\ Johns Hopkins University \\ 3400 N. Charles Street, 404 Krieger Hall \\ Baltimore, MD 21218, USA \\ ${ }^{2}$ Institute of Mathematics \\ The Hebrew University \\ 91904 Jerusalem, ISRAEL \\ and \\ ${ }^{3}$ Department of Mathematics \\ California Institute of Technology \\ Pasadena, CA 91125, USA
}

September 18, 2000

\begin{abstract}
Let $\mathbb{Z}_{+}^{d+1}=\mathbb{Z}^{d} \times \mathbb{Z}_{+}$, let $H_{0}$ be the discrete Laplacian on the Hilbert space $l^{2}\left(\mathbb{Z}_{+}^{d+1}\right)$ with a Dirichlet boundary condition, and let $V$ be a potential supported on the boundary $\partial \mathbb{Z}_{+}^{d+1}$. We introduce the notions of surface states and surface spectrum of the operator $H=H_{0}+V$ and explore their properties. Our main result is that if the potential $V$ is random and if the disorder is either large or small enough, then in dimension two $H$ has no surface spectrum on $\sigma\left(H_{0}\right)$ with probability one. To prove this result we combine Aizenman-Molchanov theory with techniques of scattering theory.
\end{abstract}

${ }^{*}$ On leave from Department of Mathematics and Statistics, University of Ottawa, 585 King Edward Avenue, Ottawa, ON, K1N 6N5, Canada 


\section{Introduction}

This paper is a direct continuation of [JL1] and deals with the following model.

Let $d \geq 1$ be given, and let $\mathbb{Z}_{+}^{d+1}=\mathbb{Z}^{d} \times \mathbb{Z}_{+}$, where $\mathbb{Z}_{+}=\{0,1, \cdots\}$. We denote the points in $\mathbb{Z}_{+}^{d+1}$ by $(n, x)$, for $n \in \mathbb{Z}^{d}$ and $x \in \mathbb{Z}_{+}$. Let $H_{0}$ be the discrete (centered) Laplacian on the Hilbert space $\mathcal{H}:=l^{2}\left(\mathbb{Z}_{+}^{d+1}\right)$ with a Dirichlet boundary condition. The operator $H_{0}$ acts as

$$
\left(H_{0} \psi\right)(n, x)= \begin{cases}\sum_{\left|n-n^{\prime}\right|++\left|x-x^{\prime}\right|=1} \psi\left(n^{\prime}, x^{\prime}\right) & \text { if } x>0 \\ \psi(n, 1)+\sum_{\left|n-n^{\prime}\right|_{+}=1} \psi\left(n^{\prime}, 0\right) & \text { if } x=0,\end{cases}
$$

where $|n|_{+}=\sum_{j=1}^{d}\left|n_{j}\right|$. Let $V$ be a potential supported on the boundary $\partial \mathbb{Z}_{+}^{d+1}=\mathbb{Z}^{d}$ (that is, $V$ acts as $(V \psi)(n, x)=V(n, x) \psi(n, x)$ and $V(n, x)=0$ if $x>0)$ and

$$
H=H_{0}+V .
$$

The model (1.1) and the questions we will study are motivated by the physics of disordered surfaces (see [JMP1, KP, P]).

In the first part of the paper we propose a dynamical definition of the surface states and surface spectrum of the operator $H$. We remark that there are some alternative approaches in the literature (see, e.g., [JMP1, DS]) and they will be compared with our proposal in [JMP2].

In the second part of the paper we consider the case where the boundary potential $V$ is a random process on $\mathbb{Z}^{d}$. This case is of particular physical importance. Our main result is that if $d+1=2$ and if the disorder is either large or small enough, then with probability one $H$ has no surface spectra on $\sigma\left(H_{0}\right)$. It is known that under these conditions the spectrum of $H$ outside $\sigma\left(H_{0}\right)$ is (with probability one) pure point with exponentially decaying eigenfunctions [JM2]. Thus, in particular, our result rules out the existence of propagating surface states in dimension two if the disorder is large or small enough.

The proof of our main result (Theorems 1.4 and 1.5 below) combines Aizenman-Molchanov theory $[\mathrm{AM}]$ with techniques of scattering theory. More precisely, we use AizenmanMolchanov theory to prove a "localization" estimate for matrix elements of the resolvent $(H-z)^{-1}$ along the boundary $\partial \mathbb{Z}_{+}^{d+1}$. Such an estimate implies that propagation of wave packets along the boundary is suppressed. We then combine this estimate with techniques of scattering theory to show that wave packets with energies in $\sigma\left(H_{0}\right)$ must dissolve into the bulk and that there are thus no surface spectra on $\sigma\left(H_{0}\right)$. A perhaps surprising aspect of our argument is that Aizenman-Molchanov theory is used to establish a result which is in spirit opposite to "localization" of wave packets. We will comment further on this point in Section 1.2. 


\subsection{Surface states and spectra}

Surface states of the model (1.1) are wave packets which remain localized near the boundary $\partial \mathbb{Z}_{+}^{d+1}$ for all time. This heuristic description can be made mathematically rigorous as follows.

For $R \in \mathbb{Z}_{+}$we set

$$
\Gamma_{R}:=\left\{(n, x): n \in \mathbb{Z}^{d}, 0 \leq x \leq R\right\}, \quad \Gamma_{\bar{R}}:=\mathbb{Z}_{+}^{d+1} \backslash \Gamma_{R} .
$$

We denote by $\mathbf{1}_{R}, \mathbf{1}_{\bar{R}}$, the characteristic functions of the sets $\Gamma_{R}, \Gamma_{\bar{R}}$, and use the same symbols for the corresponding multiplication operators (which are orthogonal projections). Obviously, $\mathbf{1}=\mathbf{1}_{R}+\mathbf{1}_{\bar{R}}$. All the results of this section hold for an arbitrary boundary potential $V$.

We say that a vector $\psi$ is a surface state of the operator $H$ if

$$
\lim _{R \rightarrow \infty} \liminf _{T \rightarrow \infty} \frac{1}{2 T} \int_{-T}^{T}\left\|\mathbf{1}_{R} \mathrm{e}^{-\mathrm{i} t H} \psi\right\|^{2} \mathrm{~d} t=\|\psi\|^{2} .
$$

We denote the set of all surface states by $\mathcal{H}_{\mathrm{s}}(H)$. Condition (1.2) is equivalent to

$$
\lim _{R \rightarrow \infty} \limsup _{T \rightarrow \infty} \frac{1}{2 T} \int_{-T}^{T}\left\|\mathbf{1}_{\bar{R}} \mathrm{e}^{-\mathrm{i} t H} \psi\right\|^{2} \mathrm{~d} t=0
$$

and from (1.3) it follows easily that $\mathcal{H}_{\mathrm{s}}(H)$ is a closed subspace of $\mathcal{H}$ invariant under $H$.

Notation. In the sequel we denote by $\mathbf{1}_{\Theta}(H)$ the spectral projection of $H$ onto a Borel set $\Theta \subset \mathbb{R}$. We will also use the shorthand $c_{d}=2(d+1)$, so $\sigma\left(H_{0}\right)=\left[-c_{d}, c_{d}\right]$.

We recall that for any $V,\left[-c_{d}, c_{d}\right] \subset \sigma(H)$ (see [JL1]). The basic property of the set $\mathcal{H}_{\mathrm{s}}(H)$ is:

Theorem 1.1 $\operatorname{Ran} \mathbf{1}_{\mathbb{R} \backslash\left(-c_{d}, c_{d}\right)}(H) \subset \mathcal{H}_{\mathrm{s}}(H)$.

The surface spectrum of the operator $H, \sigma_{\mathrm{s}}(H)$, is defined by

$$
\sigma_{\mathrm{s}}(H)=\sigma\left(\left.H\right|_{\mathcal{H}_{\mathrm{s}}}\right) \text {. }
$$

Obviously, $\sigma_{\mathrm{s}}(H)$ is a closed set and $\sigma_{\mathrm{s}}(H) \subset \sigma(H)$. Theorem 1.1 yields that

$$
\overline{\sigma(H) \backslash \sigma\left(H_{0}\right)} \subset \sigma_{\mathrm{s}}(H) \text {. }
$$

An important question is whether or not $H$ has some surface spectra on $\sigma\left(H_{0}\right)$. The only known examples where this happens are periodic potentials and some special boundary 
potentials for which $H$ has embedded eigenvalues in $\sigma\left(H_{0}\right)$ [MV]. In this paper we will describe criteria under which $H$ has no surface spectra on $\sigma\left(H_{0}\right)$.

In [CS, JL1] it was proven that for any boundary potential $V$, the wave operators

$$
\Omega^{ \pm}=\mathrm{s}-\lim _{t \rightarrow \mp \infty} \mathrm{e}^{\mathrm{i} t H} \mathrm{e}^{-\mathrm{i} t H_{0}}
$$

exist. Our next results identifies their ranges. We adopt the shorthand $\langle t\rangle=\left(1+t^{2}\right)^{\frac{1}{2}}$. Let

$$
\begin{aligned}
\mathcal{T} & :=\left\{\psi: \forall R, k \geq 0,\left\|\mathbf{1}_{R} \mathrm{e}^{-\mathrm{i} t H} \psi\right\|=O\left(\langle t\rangle^{-k}\right)\right\} \\
\mathcal{F} & :=\left\{\psi: \forall R \geq 0, \int_{\mathbb{R}}\left\|\mathbf{1}_{R} \mathrm{e}^{-\mathrm{i} t H} \psi\right\|^{2} \mathrm{~d} t<\infty\right\} .
\end{aligned}
$$

Clearly, $\mathcal{T}$ and $\mathcal{F}$ are linear spaces and $\mathcal{T} \subset \mathcal{F}$.

Theorem 1.2 $\operatorname{Ran} \Omega^{ \pm}=\overline{\mathcal{T}}=\overline{\mathcal{F}}$. Moreover, $\operatorname{Ran} \Omega^{ \pm} \subset \mathcal{H}_{\mathrm{s}}(H)^{\perp}$.

We recall that the wave operators $\Omega^{ \pm}$are complete on a Borel set $\Theta \subset \mathbb{R}$ if $\operatorname{Ran} \mathbf{1}_{\Theta}(H) \subset$ $\operatorname{Ran} \Omega^{ \pm}$. Therefore, Theorem 1.2 yields:

Corollary 1.3 If the wave operators $\Omega^{ \pm}$are complete on $\Theta$, then $\sigma_{\mathrm{s}}(H) \cap \Theta=\emptyset$.

In [JL1] we have introduced the notion of resonant spectrum, $\mathfrak{R}(H)$, and we have shown that the wave operators $\Omega^{ \pm}$are complete on $\sigma(H) \backslash \mathfrak{R}(H)$. Thus, $\sigma_{\mathrm{s}}(H) \subset \mathfrak{R}(H)$. Various estimates on the location of the set $\mathfrak{R}(H)$ are given in [JL1].

\subsection{Random boundary potentials}

Let $\Omega$ be the set of all boundary potentials,

$$
\Omega=\mathbb{R}^{\mathbb{Z}^{d}}=\underset{\mathbb{Z}^{d}}{X} \mathbb{R}
$$

let $\mathfrak{B}$ be the Borel $\sigma$-algebra in $\Omega$ and $\mathrm{d} P$ a probability measure on $(\Omega, \mathfrak{B})$ of the form

$$
\mathrm{d} P=\underset{\mathbb{Z}^{d}}{X} \mathrm{~d} \mu,
$$

where $\mathrm{d} \mu=p(x) \mathrm{d} x$ is an absolutely continuous (w.r.t. Lebesgue measure) probability measure on $\mathbb{R}$. In what follows we use the symbol $\mathbb{E}(f)$ for the expectation of a random variable $f$ and assume that $d+1=2$.

The main result of this paper is: 
Theorem 1.4 Let $U_{\text {per }}$ be a periodic boundary potential and $H=H_{0}+U_{\mathrm{per}}+\lambda V, V \in \Omega$, where $\lambda$ is a real constant. Assume that $\langle x\rangle^{\alpha} p(x) \in L^{1}(\mathbb{R}) \cap L^{\infty}(\mathbb{R})$ for some $\alpha>2 / 3$. Then there is a constant $\Lambda>0$ such that for $|\lambda|>\Lambda, R \geq 0$ and $\psi \in \mathcal{H}$,

$$
\lim _{T \rightarrow \infty} \frac{1}{2 T} \int_{-T}^{T} \mathbb{E}\left(\left\|\mathbf{1}_{R} \mathrm{e}^{-\mathrm{i} t H} \mathbf{1}_{\left(-c_{d}, c_{d}\right)}(H) \psi\right\|^{2}\right) \mathrm{d} t=0 .
$$

In particular, for $|\lambda|>\Lambda$,

$$
\sigma_{\mathrm{s}}(H) \cap\left(-c_{d}, c_{d}\right)=\emptyset \quad P-a . s .
$$

Remark 1. That (1.5) implies (1.6) is seen as follows. Let $\mathcal{N}$ be a countable, dense set in $\mathcal{H}$. Fatou's lemma and (1.5) yield that there is a set $\tilde{\Omega} \subset \Omega$ of full $P$-measure such that for $V \in \tilde{\Omega}$ and $\psi \in \mathcal{N}$

$$
\lim _{R \rightarrow \infty} \liminf _{T \rightarrow \infty} \frac{1}{2 T} \int_{-T}^{T}\left\|\mathbf{1}_{R} \mathrm{e}^{-\mathrm{i} t H} \mathbf{1}_{\left(-c_{d}, c_{d}\right)}(H) \psi\right\|^{2} \mathrm{~d} t=0 .
$$

Since for fixed $V$ the set of $\psi$ 's for which (1.7) holds is closed, (1.7) holds for $V \in \tilde{\Omega}$ and $\psi \in \overline{\mathcal{N}}=\mathcal{H}$.

Remark 2. The constant $|\lambda|$ is a measure of the disorder.

Remark 3. The operator $H_{0}+U_{\text {per }}$ may have some surface spectra on $\sigma\left(H_{0}\right)$. For example, if $\forall n, U_{\text {per }}(n, 0)=a$ and $|a|>1$, then $\sigma_{\mathrm{s}}\left(H_{0}+U_{\text {per }}\right)=[-2 d, 2 d]+a+a^{-1}$. One can also show that this surface spectrum is stable under short-range perturbations, see [JMP2].

Remark 4. The technical conditions of Theorem 1.4 can be relaxed in several ways. The method of the proof allows for more general densities (non-i.i.d. and correlated random variables), the background potential does not have to be periodic, etc. We opted for conditions which cover most physically interesting situations, while allowing for a technically simple exposition of the results and the proofs.

If the background potential $U_{\text {per }}$ is equal to zero, then we can also deal with the weak coupling regime.

Theorem 1.5 Let $H=H_{0}+\lambda V, V \in \Omega$, and assume that $\langle x\rangle^{\alpha} p(x)$ is in $L^{1}(\mathbb{R})$ for some $\alpha>2 / 3$ and in $L^{\infty}(\mathbb{R})$ for some $\alpha>5 / 3$. Then there is a constant $\Lambda>0$ such that for $|\lambda|<\Lambda, R \geq 0$ and $\psi \in \mathcal{H}$,

$$
\lim _{T \rightarrow \infty} \frac{1}{2 T} \int_{-T}^{T} \mathbb{E}\left(\left\|\mathbf{1}_{R} \mathrm{e}^{-\mathrm{i} t H} \mathbf{1}_{\left(-c_{d}, c_{d}\right)}(H) \psi\right\|^{2}\right) \mathrm{d} t=0 .
$$

In particular, for $|\lambda|<\Lambda, \sigma_{\mathrm{s}}(H) \cap\left(-c_{d}, c_{d}\right)=\emptyset P$-a.s. 
Remark 1. If $\Lambda\|V\| \leq 1 P$-a.s., then for $|\lambda|<\Lambda$, the wave operators (1.4) are complete on $\sigma\left(H_{0}\right) P$-a.s. [JL1], and by Corollary $1.3 H$ has $P$-a.s. no surface spectra on $\sigma\left(H_{0}\right)$. Thus, for bounded random variables the above theorem could be an empty statement. If, however, $\operatorname{supp} \mu=\mathbb{R}$ (for example, the random variables $\{V(n)\}$ have a Cauchy or Gaussian distribution), then it is not known whether the wave operators (1.4) are complete for any $\lambda \neq 0$. In such situations, Theorem 1.5 is a new result.

Remark 2. The cases $U_{\text {per }}=0$ and $U_{\text {per }} \neq 0$ may describe two very different physical situations. See [JM2, JM3] for a discussion.

We finish this section with a brief discussion of Theorems 1.4 and 1.5.

Roughly, the physical reason why these theorems hold is that in the strong coupling regime (with $U_{\text {per }}$ possibly different from zero) and in the weak coupling regime (with $\left.U_{\text {per }}=0\right)$ the propagation of wave packets along the boundary is suppressed. This forces wave packets with energies outside $\sigma\left(H_{0}\right)$ to turn into bound states, which is the reason why we have only pure point spectrum outside $\sigma\left(H_{0}\right)$ in these regimes [JM1, JM2]. However, since the spectrum of $H$ in $\sigma\left(H_{0}\right)$ is $P$-a.s. purely a.c. [JL1, JL2], wave packets with energies in that regime must propagate, and they are thus expelled into the bulk.

Notation. Throughout the paper we will use the shorthands

$$
\begin{gathered}
\mathcal{R}(z):=(H-z)^{-1}, \\
\mathcal{R}((m, y),(n, x) ; z):=\left(\delta_{(m, y)} \mid(H-z)^{-1} \delta_{(n, x)}\right) .
\end{gathered}
$$

The above heuristic argument can be made mathematically rigorous as follows. Let $(a, b)$ be given energy interval and $0<s<1$. For $m, n \in \mathbb{Z}^{d}$ and $x \in \mathbb{Z}_{+}$let

$$
\gamma_{x}(m, n):=\sup _{e \in(a, b), \epsilon \neq 0} \mathbb{E}\left(|\mathcal{R}((m, 0),(n, x), e+\mathrm{i} \epsilon)|^{s}\right) .
$$

The estimate one seeks is that for all $x \geq 0$ there are positive constants $C$ and $\delta$ such that $\forall m, n$,

$$
\gamma_{x}(m, n) \leq C\langle n-m\rangle^{-d-\delta} .
$$

If $(a, b) \cap \sigma\left(H_{0}\right)=\emptyset$, then (1.8) implies that on $(a, b)$ the operator $H$ has $P$-a.s. only pure point spectrum [JM2]. (For this result it suffices that (1.8) holds for $x=0$ ). On the other hand, if $(a, b) \subset \sigma\left(H_{0}\right)$, then (1.8) implies that relation (1.5) holds and that $H$ has $P$-a.s. no surface spectra on $(a, b)$ (see Section 3.2).

The question remains how the estimate (1.8) can be established. Let us consider the case $x=0$. One can show that for any $z \in \mathbb{C} \backslash \sigma\left(H_{0}\right)$ there exists an operator $h_{0}(z)$, which 
acts on $l^{2}\left(\mathbb{Z}^{d}\right)$ as convolution by a sequence $j(n, z)$,

$$
\left(h_{0}(z) \psi\right)(n)=\sum_{k} j(n-k, z) \psi(k),
$$

so that

$$
\left(\delta_{(m, 0)} \mid(H-z)^{-1} \delta_{(n, 0)}\right)=\left(\delta_{m} \mid(h(z)-z)^{-1} \delta_{n}\right),
$$

where $h(z)=h_{0}(z)+V$. Thus, the estimate (1.8) for $x=0$ is the usual localization estimate of Aizenman-Molchanov [AM] for the operator $h(z)$. The difficulties in establishing such an estimate stem from the fact that $h_{0}(z)$ is a long-range energy-dependent Laplacian, and the existing techniques are effective only if for some $\delta>0$ and $\forall n$

$$
t(n):=\sup _{e \in(a, b), \epsilon \neq 0}|j(n, e+\mathrm{i} \epsilon)|=O\left(\langle n\rangle^{-d-\delta}\right) .
$$

However, it turns out that $t(n) \sim\langle n\rangle^{-(d+2) / 2}$, and we have an appropriate decay only if $d+1=2$. This is the reason why we are able to prove Theorems 1.4 and 1.5 only in dimension two. We do not know whether this restriction is technical or new physical phenomena emerge in dimensions $d+1>2$.

Acknowledgments. We would like to thank S. Molchanov, L. Pastur, B. Simon and B. Vainberg for useful discussions and to A. Kelm for comments on the manuscript. VJ's work was partially supported by NSERC. YL's work was partially supported by NSF grant DMS-9801474, by an ISF grant, and by an Allon fellowship.

\section{Deterministic results}

\subsection{Proof of Theorem 1.1}

The following result will be used on several occasions in this paper.

Lemma 2.1 Let $A$ be a bounded self-adjoint operator on $\mathcal{H}$. Then, for any $\psi \in \mathcal{H}$,

$$
\frac{1}{2 T} \int_{-T}^{T}\left\|A \mathrm{e}^{-\mathrm{i} t H} \psi\right\|^{2} \mathrm{~d} t \leq C \epsilon \max _{ \pm} \int_{\mathbb{R}}\|A \mathcal{R}(e \pm \mathrm{i} \epsilon) \psi\|^{2} \mathrm{~d} e,
$$

where $\epsilon=T^{-1}$ and $C=\mathrm{e}^{2} / 2 \pi$. 
Proof. The result follows from the well-known identity (see, e.g., [RS], Section XIII.7)

$$
2 \pi \int_{\mathbb{R}} \mathrm{e}^{-2 \epsilon|t|}\left\|A \mathrm{e}^{-\mathrm{i} t H} \psi\right\|^{2} \mathrm{~d} t=\int_{\mathbb{R}}\left(\|A \mathcal{R}(e+\mathrm{i} \epsilon) \psi\|^{2}+\|A \mathcal{R}(e-\mathrm{i} \epsilon) \psi\|^{2}\right) \mathrm{d} e
$$

and the estimate

$$
\frac{1}{2 T} \int_{-T}^{T}\left\|A \mathrm{e}^{-\mathrm{i} t H} \psi\right\|^{2} \mathrm{~d} t \leq \frac{\mathrm{e}^{2} \epsilon}{2} \int_{\mathbb{R}} \mathrm{e}^{-2 \epsilon|t|}\left\|A \mathrm{e}^{-\mathrm{i} t H} \psi\right\|^{2} \mathrm{~d} t
$$

where $\epsilon=T^{-1}$.

The following lemma is an immediate consequence of Proposition 3.1 of [JL1]. We use the shorthand $\mathcal{R}_{0}(z)=\left(H_{0}-z\right)^{-1}$.

Lemma 2.2 If $[a, b] \cap \sigma\left(H_{0}\right)=\emptyset$ then there exists $\gamma>0$ such that

$$
\sup _{e \in[a, b], \epsilon \neq 0}\left\|\mathbf{1}_{\bar{R}} \mathcal{R}_{0}(e+\mathrm{i} \epsilon) \mathbf{1}_{0}\right\| \leq C \mathrm{e}^{-\gamma R} .
$$

Lemma 2.3 Let $\Theta$ be a compact set such that $\Theta \cap \sigma\left(H_{0}\right)=\emptyset$. Then there exist constants $C>0$ and $\gamma>0$ such that for all $R \geq 0$ and $n \in \mathbb{Z}^{d}$,

$$
\limsup _{\epsilon \rightarrow 0}|\epsilon| \int_{\mathbb{R}}\left\|\mathbf{1}_{\bar{R}} \mathcal{R}(e+\mathrm{i} \epsilon) \mathbf{1}_{\Theta}(H) \delta_{(n, 0)}\right\|^{2} \mathrm{~d} e \leq C \mathrm{e}^{-\gamma R} .
$$

Proof. Let $[a, b]$ be an interval such that $\Theta$ is properly contained in $[a, b]$ and $[a, b] \cap$ $\sigma\left(H_{0}\right)=\emptyset$. Then there exists a constant $C_{1}$ such that $\forall e \in \mathbb{R} \backslash[a, b]$,

$$
\sup _{\epsilon}\left\|\mathcal{R}(e+\mathrm{i} \epsilon) \mathbf{1}_{\Theta}(H)\right\| \leq C_{1} / \operatorname{dist}(e, \Theta) .
$$

Thus, it suffices to show that

$$
\limsup _{\epsilon \rightarrow 0}|\epsilon| \int_{a}^{b}\left\|\mathbf{1}_{\bar{R}} \mathcal{R}(e+\mathrm{i} \epsilon) \mathbf{1}_{\Theta}(H) \delta_{(n, 0)}\right\|^{2} \mathrm{~d} e \leq C \mathrm{e}^{-\gamma R} .
$$

Since $V=\mathbf{1}_{0} V$ and

$$
\sup _{\epsilon \neq 0}|\epsilon| \int_{a}^{b}\left\|V \mathcal{R}(e+\mathrm{i} \epsilon) \mathbf{1}_{\Theta}(H) \delta_{(n, 0)}\right\|^{2} \leq C_{2},
$$

the result follows from the resolvent identity and Lemma 2.2. 
We are now ready to finish:

Proof of Theorem 1.1. Let $\Theta$ be a compact set such that $\Theta \cap \sigma\left(H_{0}\right)=\emptyset$. It follows from Lemmas 2.1 and 2.3 that for any $n \in \mathbb{Z}^{d}$,

$$
\limsup _{T \rightarrow \infty} \frac{1}{2 T} \int_{-T}^{T}\left\|\mathbf{1}_{\bar{R}} \mathrm{e}^{-\mathrm{i} t H} \mathbf{1}_{\Theta}(H) \delta_{(n, 0)}\right\|^{2} \mathrm{~d} t=O\left(\mathrm{e}^{-\gamma R}\right) .
$$

Thus, for all $n \in \mathbb{Z}^{\mathrm{d}}, \mathbf{1}_{\Theta}(H) \delta_{(n, 0)} \subset \mathcal{H}_{\mathrm{s}}(H)$. Since the set $\left\{\delta_{(n, 0)}: n \in \mathbb{Z}^{d}\right\}$ is cyclic for $H$ (see [JL1]), we have that $\operatorname{Ran} \mathbf{1}_{\mathbb{R} \backslash\left[-c_{d}, c_{d}\right]}(H) \subset \mathcal{H}_{\mathrm{s}}(H)$. Finally, either $\mathbf{1}_{\left\{ \pm c_{d}\right\}}(H)=0$ or $\pm c_{d}$ is an eigenvalue of $H$. In either case, $\operatorname{Ran} \mathbf{1}_{\left\{ \pm c_{d}\right\}}(H) \subset \mathcal{H}_{\mathrm{s}}(H)$ and Theorem 1.1 follows.

\section{$2.2 \quad$ Ranges of $\Omega^{ \pm}$}

In this section we prove Theorem 1.2.

Let

$$
\mathcal{D}:=\left\{\psi: \forall R, k \geq 0,\left\|\mathbf{1}_{R} \mathrm{e}^{-\mathrm{i} t H_{0}} \psi\right\|=O\left(\langle t\rangle^{-k}\right)\right\} .
$$

Obviously, $\mathcal{D}$ is a linear subspace of $\mathcal{H}$. Moreover, in [JL1] (Proposition 3.12) we have shown that

$$
\overline{\mathcal{D}}=\mathcal{H}
$$

Theorem 1.2 follows from the next three propositions.

Proposition 2.4 $\operatorname{Ran} \Omega^{ \pm} \subset \overline{\mathcal{T}}$.

Proof. We consider the - case; a similar argument applies to the + case.

Let $T$ be a linear operator defined by

$$
T \delta_{(n, x)}=\left\{\begin{array}{cc}
-\delta_{(n, 1)} & \text { if } x=0 \\
\delta_{(n, 0)} & \text { if } x=1 \\
0 & \text { if } x>1
\end{array}\right.
$$

Note that $\|T\|=1$ and

$$
H \mathbf{1}_{\overline{0}}-\mathbf{1}_{\overline{0}} H_{0}=\left[H_{0}, \mathbf{1}_{\overline{0}}\right]=T .
$$


Fix $\psi \in \mathcal{D}$. Since

$$
\lim _{t \rightarrow \infty} \mathrm{e}^{\mathrm{i} t H} \mathbf{1}_{0} \mathrm{e}^{-\mathrm{i} t H_{0}} \psi=0
$$

we have

$$
\begin{aligned}
\Omega^{-} \psi-\psi & =\int_{0}^{\infty} \frac{\mathrm{d}}{\mathrm{d} \tau} \mathrm{e}^{\mathrm{i} \tau H} \mathbf{1}_{\overline{0}} \mathrm{e}^{-\mathrm{i} \tau H_{0}} \psi \mathrm{d} \tau \\
& =\mathrm{i} \int_{0}^{\infty} \mathrm{e}^{\mathrm{i} \tau H} T \mathrm{e}^{-\mathrm{i} \tau H_{0}} \psi \mathrm{d} \tau
\end{aligned}
$$

Thus,

$$
\begin{aligned}
\left\|\mathbf{1}_{R} \mathrm{e}^{-\mathrm{i} t H} \Omega^{-} \psi\right\|^{2} & =\left\|\mathbf{1}_{R} \Omega^{-} \mathrm{e}^{-\mathrm{i} t H_{0}} \psi\right\|^{2} \\
& \leq A(t)+2\left\|\mathbf{1}_{R} \mathrm{e}^{-i t H_{0}} \psi\right\|^{2}
\end{aligned}
$$

where

$$
A(t)=2\left(\int_{0}^{\infty}\left\|T \mathrm{e}^{-\mathrm{i}(\tau+t) H_{0}} \psi\right\| \mathrm{d} \tau\right)^{2} .
$$

Since $T=T \mathbf{1}_{1}$, it follows from the definition of $\mathcal{D}$ and (2.12) that for any $\psi \in \mathcal{D}, R \geq 0$ and $k \geq 0$,

$$
\left\|\mathbf{1}_{R} \mathrm{e}^{-\mathrm{i} t H} \Omega^{-} \psi\right\|^{2}=O\left(\langle t\rangle^{-k}\right) .
$$

Thus, $\Omega^{-} \mathcal{D} \subset \mathcal{T}$, and relation (2.9) yields the result.

Proposition 2.5 $\overline{\mathcal{F}} \subset \operatorname{Ran} \Omega^{ \pm}$.

Proof. We again consider only the - case. Obviously, to prove the statement it suffices to show that for every $\psi \in \mathcal{F}$ the limit

$$
\lim _{t \rightarrow \infty} \mathrm{e}^{\mathrm{i} t H_{0}} \mathrm{e}^{-\mathrm{i} t H} \psi
$$

exists.

First, we observe that for $\psi \in \mathcal{F}$,

$$
\lim _{t \rightarrow \infty} \mathrm{e}^{\mathrm{i} t H} \mathbf{1}_{0} \mathrm{e}^{-\mathrm{i} t H} \psi=0
$$


Indeed, let $w(t)=\mathrm{e}^{\mathrm{i} t H} \mathbf{1}_{0} \mathrm{e}^{-\mathrm{i} t H} \psi$. Then $\int\|w(t)\|^{2} \mathrm{~d} t<\infty$, and, since $\left[H, \mathbf{1}_{0}\right]=\left[H_{0}, \mathbf{1}_{0}\right]$, $\left\|w^{\prime}(t)\right\| \leq 2\left\|H_{0}\right\|$. This yields that $\lim _{t \rightarrow \infty} w(t)=0$ (see, e.g., Exercise 6.2 in [RS]). Thus, to prove the existence of the limit (2.13) it suffices to prove the existence of

$$
\lim _{t \rightarrow \infty} \mathrm{e}^{\mathrm{i} t H_{0}} \mathbf{1}_{\overline{0}} \mathrm{e}^{-\mathrm{i} t H} \psi
$$

We fix $\psi \in \mathcal{F}$ and set

$$
\ell(t):=\mathrm{e}^{\mathrm{i} t H_{0}} \mathbf{1}_{\overline{0}} \mathrm{e}^{-\mathrm{i} t H} \psi
$$

Let $\phi \in \mathcal{H}$ be arbitrary. Then

$$
\frac{\mathrm{d}}{\mathrm{d} t}(\phi \mid w(t))=\mathrm{i}\left(\mathrm{e}^{-\mathrm{i} t H_{0}} \phi \mid T \mathrm{e}^{-\mathrm{i} t H} \psi\right),
$$

where $T$ is given by (2.10). Since $T=T \mathbf{1}_{1}=\mathbf{1}_{1} T$, we have that for $t>s$,

$$
|(\phi \mid \ell(t)-\ell(s))| \leq\left(\int_{\mathbb{R}}\left\|\mathbf{1}_{1} \mathrm{e}^{-\mathrm{i} \tau H_{0}} \phi\right\|^{2} \mathrm{~d} \tau\right)^{\frac{1}{2}}\left(\int_{s}^{t}\left\|\mathbf{1}_{1} \mathrm{e}^{-\mathrm{i} \tau H} \psi\right\|^{2} \mathrm{~d} \tau\right)^{\frac{1}{2}} .
$$

Since $\mathbf{1}_{1}$ is $H_{0}$-smooth (see Lemma 3.7 in [JL1]), there is a constant $C$, independent of $\phi$, such that

$$
\left(\int_{\mathbb{R}}\left\|\mathbf{1}_{1} \mathrm{e}^{-\mathrm{i} \tau H_{0}} \phi\right\|^{2} \mathrm{~d} \tau\right)^{\frac{1}{2}} \leq C\|\phi\|
$$

Therefore,

$$
\|\ell(t)-\ell(s)\| \leq C\left(\int_{s}^{t}\left\|\mathbf{1}_{1} \mathrm{e}^{-\mathrm{i} \tau H} \psi\right\|^{2} \mathrm{~d} \tau\right)^{\frac{1}{2}}
$$

If $\psi \in \mathcal{F}$, then the integrand on the right hand side of the last equation is in $L^{1}(\mathbb{R})$. Therefore, the sequence $\ell(t)$ is Cauchy as $t \rightarrow \infty$ and $\lim _{t \rightarrow \infty} \ell(t)$ exist.

Proposition 2.6 $\overline{\mathcal{F}} \perp \mathcal{H}_{\mathrm{s}}(H)$.

Proof. Let $\phi \in \mathcal{F}$ and $\psi \in \mathcal{H}_{\mathrm{s}}(H)$ be two normalized vectors. Then

$$
(\phi \mid \psi)=\left(\mathbf{1}_{R} \mathrm{e}^{-\mathrm{i} t H} \phi \mid \mathbf{1}_{R} \mathrm{e}^{-\mathrm{i} t H} \psi\right)+\left(\mathbf{1}_{\bar{R}} \mathrm{e}^{-\mathrm{i} t H} \phi \mid \mathbf{1}_{\bar{R}} \mathrm{e}^{-\mathrm{i} t H} \psi\right),
$$

which yields

$$
|(\phi \mid \psi)| \leq\left\|\mathbf{1}_{R} \mathrm{e}^{-\mathrm{i} t H} \phi\right\|+\left\|\mathbf{1}_{\bar{R}} \mathrm{e}^{-\mathrm{i} t H} \psi\right\|
$$


Thus,

$$
|(\phi \mid \psi)| \leq\left(\frac{1}{2 T} \int_{-T}^{T}\left\|\mathbf{1}_{R} \mathrm{e}^{-\mathrm{i} t H} \phi\right\|^{2} \mathrm{~d} t\right)^{\frac{1}{2}}+\left(\frac{1}{2 T} \int_{-T}^{T}\left\|\mathbf{1}_{\bar{R}} \mathrm{e}^{-\mathrm{i} t H} \psi\right\|^{2} \mathrm{~d} t\right)^{\frac{1}{2}}
$$

and

$$
\begin{aligned}
|(\phi \mid \psi)| \leq & \limsup _{T \rightarrow \infty}\left(\frac{1}{2 T} \int_{-T}^{T}\left\|\mathbf{1}_{R} \mathrm{e}^{-\mathrm{i} t H} \phi\right\|^{2} \mathrm{~d} t\right)^{\frac{1}{2}} \\
& +\lim _{R \rightarrow \infty} \limsup _{T \rightarrow \infty}\left(\frac{1}{2 T} \int_{-T}^{T}\left\|\mathbf{1}_{\bar{R}} \mathrm{e}^{-\mathrm{i} t H} \psi\right\|^{2} \mathrm{~d} t\right)^{\frac{1}{2}}=0 .
\end{aligned}
$$

\section{Surface spectra in dimension two}

In this section we prove Theorems 1.4 and 1.5. In what follows we assume that $d+1=2$. We use the letter $C$ for a generic constant which may change from estimate to estimate.

\subsection{Preliminaries}

In this section we collect some technical results about the model (1.1). Most of them are similar to the results already discussed in [JL1, JM1, JM2, JM3] and we refer the reader to any of these papers for additional information. All the results of this section, except for Lemma 3.4, hold for an arbitrary boundary potential $V$.

Let $\mathbb{T}$ be the unit circle. We denote by $\phi$ the points in $\mathbb{T}$ and by $\mathrm{d} \phi$ the usual Lebesgue measure. Let $\mathbb{C}_{ \pm}=\{z: \pm \operatorname{Im} z>0\}$ and, for $z \in \mathbb{C}_{ \pm}$, let $\lambda(\phi, z)$ be the root of the quadratic equation

$$
X+X^{-1}+2 \cos \phi=z
$$

which satisfies $|\lambda(\phi, z)|<1$. Explicitly, for $z \in \mathbb{C}_{ \pm}$,

$$
\lambda(\phi, z)=\frac{1}{2}\left(2 \cos \phi-z-\sqrt{(2 \cos \phi-z)^{2}-4_{ \pm}}\right)
$$

where the branch of square root is fixed by

$$
\sqrt{w}_{ \pm}=\sqrt{x+\mathrm{i} y_{ \pm}}=\frac{\sqrt{2}}{2}(\sqrt{|w|+x} \pm \mathrm{i} \sqrt{|w|-x}), \quad \pm \operatorname{Im} w>0 .
$$

The function $\lambda(\phi, z)$ extends by continuity from $\mathbb{T} \times \mathbb{C}_{ \pm}$to $\mathbb{T} \times \overline{\mathbb{C}}_{ \pm}$. We denote the values of these extensions along the real axis by $\lambda(\phi, e \pm \mathrm{i} 0)$. 
Lemma 3.1 For any $x \in \mathbb{Z}_{+}$there is a constant $C_{x}$ such that $\forall n$,

$$
\sup _{z \in \mathbb{C}_{ \pm}}\left|\int_{\mathbb{T}} \mathrm{e}^{-\mathrm{i} n \phi} \lambda(\phi, z)^{x} \mathrm{~d} \phi\right| \leq C_{x}\langle n\rangle^{-3 / 2} .
$$

Proof. In [JL1] we have shown that

$$
\left(\delta_{(0,0)} \mid\left(H_{0}-z\right)^{-1} \delta_{(n, x)}\right)=-(2 \pi)^{-1 / 2} \int_{\mathbb{T}} \mathrm{e}^{-\mathrm{i} n \phi} \lambda(\phi, z)^{x+1} \mathrm{~d} \phi .
$$

Moreover, the proof of Lemma 2.9 in [JM2] yields that for some $D_{x}$ and all $n$,

$$
\sup _{|e|>4}\left|\int_{\mathbb{T}} \mathrm{e}^{-\mathrm{i} n \phi} \lambda(\phi, e)^{x} \mathrm{~d} \phi\right| \leq D_{x}\langle n\rangle^{-2} .
$$

Thus, the maximum modulus principle and the standard exponential estimate on the Green function (3.17) yield that (3.16) follows if for some $C_{x}$ and all $n$

$$
\sup _{|e| \leq 4}\left|\int_{\mathbb{T}} \mathrm{e}^{-\mathrm{i} n \phi} \lambda(\phi, e \pm \mathrm{i} 0)^{x} \mathrm{~d} \phi\right| \leq C_{x}\langle n\rangle^{-3 / 2} .
$$

The proof of this fact is a calculus exercise. We indicate below the main steps of this calculation.

For notational simplicity, we consider the cases $x=1,+\mathrm{i} 0$, and $e \geq 0$. We need to estimate the integral

$$
I_{n}=\int_{\mathbb{T}} \mathrm{e}^{-\mathrm{i} n \phi} \sqrt{(2 \cos \phi-e)^{2}-4}+\mathrm{d} \phi
$$

for $e \in[0,4]$ and $|n| \gg 1$. The change of variable $u=\cos \phi$ and integration by parts yield that

$$
\left|I_{n}\right| \leq\left(A_{n+}+A_{n-}\right) /|n|
$$

where

$$
A_{n \pm}=\left|\int_{-1}^{1} \mathrm{e}^{-\mathrm{i} n \cos ^{-1} u} \frac{2(2 u \pm e)}{\sqrt{(2 u \pm e)^{2}-4}} \mathrm{~d} u\right| .
$$

We estimate $A_{n+}$. Let $0<\delta \ll 1$ and

$$
\mathcal{S}(\delta)=\{u \in[-1,1]: \operatorname{dist}(u, \pm 1-e / 2) \leq \delta\}
$$


Let $\Psi_{n+}$ be the integrand in $A_{n+}$. One can easily see that

$$
\left|\int_{\mathcal{S}(\delta)} \Psi_{n+}(u) \mathrm{d} u\right| \leq C \sqrt{\delta}
$$

If $\mathcal{S}^{\mathrm{c}}(\delta)=[-1,1] \backslash \mathcal{S}(\delta)$, then

$$
\left|\int_{\mathcal{S}^{\mathrm{c}}(\delta)} \Psi_{n+}(u) \mathrm{d} u\right|=\frac{1}{|n|}\left|\int_{\mathcal{S}^{\mathrm{c}}(\delta)}\left(\frac{d}{d u} \mathrm{e}^{-\mathrm{i} n \cos ^{-1} u}\right) \sqrt{1-u^{2}} \frac{2(2 u+e)}{\sqrt{(2 u+e)^{2}-4}} \mathrm{~d} u\right|,
$$

and integration by parts yields

$$
\left|\int_{\mathcal{S}^{\mathrm{c}}(\delta)} \Psi_{n+}(u) \mathrm{d} u\right| \leq \frac{C}{|n| \sqrt{\delta}}
$$

Therefore, for some constant $C$ independent of $e$,

$$
\left|I_{n}\right| \leq \frac{C}{|n|}\left(\sqrt{\delta}+\frac{1}{|n| \sqrt{\delta}}\right)
$$

Setting $\delta=|n|^{-1}$, we derive the statement.

Let

$$
j(n, z)=(2 \pi)^{-1} \int_{\mathbb{T}} \mathrm{e}^{-\mathrm{i} n \phi}(\lambda(\phi, z)+2 \cos \phi) \mathrm{d} \phi,
$$

and let $h_{0}(z)$ be the operator of convolution by $j(n, z)$. This operator acts on $l^{2}(\mathbb{Z})$ as follows:

$$
\left(h_{0}(z) \psi\right)(n)=\sum_{k} j(n-k, z) \psi(k)
$$

Let $h(z)=h_{0}(z)+V$ and $H=H_{0}+V$, where $V$ is a boundary potential. Note that $h(z)$ acts on $l^{2}(\mathbb{Z})$ while $H$ acts on $l^{2}\left(\mathbb{Z}_{+}^{2}\right)$. Let

$$
\hat{\mathcal{R}}((m, 0),(\phi, x) ; z)=(2 \pi)^{-\frac{1}{2}} \sum_{n} \mathcal{R}((m, 0),(n, x) ; z) \mathrm{e}^{\mathrm{i} n \phi} .
$$

For the proof of the next two lemmas we refer the reader to [JL1, JM1].

Lemma 3.2 For any $n, m \in \mathbb{Z}$ and $z \in \mathbb{C}_{ \pm}$,

$$
\left(\delta_{(m, 0)} \mid(H-z)^{-1} \delta_{(n, 0)}\right)=\left(\delta_{m} \mid(h(z)-z)^{-1} \delta_{n}\right) .
$$


Lemma 3.3 For any $x \in \mathbb{Z}_{+}$and $z \in \mathbb{C}_{ \pm}$,

$$
\hat{\mathcal{R}}((m, 0),(\phi, x), z)=\hat{\mathcal{R}}((m, 0),(\phi, 0) ; z) \lambda(\phi, z)^{x} .
$$

Our next lemma deals with a random potential $V$. Let $(\Omega, \mathfrak{B})$ be as in Section 1.2 and $P$ be an arbitrary probability measure on $(\Omega, \mathfrak{B})$.

Lemma 3.4 Let $(a, b)$ be an interval such that for some $2 / 3<s<1, \delta>0$ and all $m, n \in \mathbb{Z}$,

$$
\sup _{e \in(a, b), \epsilon \neq 0} \mathbb{E}\left(|\mathcal{R}((m, 0),(n, 0) ; e+\mathrm{i} \epsilon)|^{s}\right)<C\langle n-m\rangle^{-1-\delta} .
$$

Then for all $x \in \mathbb{Z}_{+}$there is a constant $C_{x}$ such that

$$
\sup _{e \in(a, b), \epsilon \neq 0} \mathbb{E}\left(|\mathcal{R}((m, 0),(n, x) ; e+\mathrm{i} \epsilon)|^{s}\right)<C_{x}\langle n-m\rangle^{-q},
$$

where $q=\min (1+\delta, 3 s / 2)$.

Proof. Let

$$
t_{x}(n, z)=(2 \pi)^{-1} \int_{\mathbb{T}} \mathrm{e}^{-\mathrm{i} n \phi} \lambda(\phi, z)^{x} \mathrm{~d} \phi
$$

Then by Lemma 3.3,

$$
\mathcal{R}((m, 0),(n, x) ; z)=\sum_{k} t_{x}(n-k, z) \mathcal{R}((m, 0),(k, 0) ; z),
$$

and it follows that

$$
\mathbb{E}\left(|\mathcal{R}((m, 0),(n, x) ; z)|^{s}\right) \leq \sum_{k}\left|t_{x}(n-k, z)\right|^{s} \mathbb{E}\left(|\mathcal{R}((m, 0),(k, 0) ; z)|^{s}\right) .
$$

The result now follows from Lemma 3.1 and (3.19).

The last result we will need is:

Lemma 3.5 Let $\delta>0$ be given. Then there is a constant $C$ such that for $l>0$ and $m, n \in \mathbb{Z}$

$$
\sum_{k_{1}, \cdots, k_{l} \in \mathbb{Z}}\left(\left\langle k_{1}-m\right\rangle\left\langle k_{2}-k_{1}\right\rangle \ldots\left\langle k_{l}-k_{l-1}\right\rangle\left\langle n-k_{l}\right\rangle\right)^{-1-\delta} \leq C^{l}\langle n-m\rangle^{-1-\delta} .
$$

Proof. An elementary induction. 


\subsection{The property $\mathcal{P}$}

Throughout this section we assume that the conditions of Theorem 1.4 hold.

Let $(a, b) \subset\left(-c_{d}, c_{d}\right)$. We say that the property $\mathcal{P}$ holds on $(a, b)$ if for all $R \geq 0$ and $\psi \in \mathcal{H}$,

$$
\lim _{T \rightarrow \infty} \frac{1}{2 T} \int_{-T}^{T} \mathbb{E}\left(\left\|\mathbf{1}_{R} \mathrm{e}^{-\mathrm{i} t H} \mathbf{1}_{(a, b)}(H) \psi\right\|^{2}\right) \mathrm{d} t=0 .
$$

To prove Theorem 1.4 it suffices to show that the property $\mathcal{P}$ holds on every interval $(a, b)$ properly contained in $\left(-c_{d}, c_{d}\right)$.

In what follows we fix an interval $(a, b)$ properly contained in $\left(-c_{d}, c_{d}\right)$ and $s$ such that $2 / 3<s<1$ and $\langle x\rangle^{s} p(x) \in L^{1}(\mathbb{R}) \cap L^{\infty}(\mathbb{R})$. The goal of this section is to prove

Theorem 3.6 Assume that for some $\delta>0$ and all $m, n \in \mathbb{Z}$,

$$
\sup _{e \in(a, b), \epsilon \neq 0} \mathbb{E}\left(|\mathcal{R}((m, 0),(n, 0), e+\mathrm{i} \epsilon)|^{s}\right) \leq C\langle n-m\rangle^{-1-\delta} .
$$

Then the property $\mathcal{P}$ holds on $(a, b)$.

First, we need the following result of Graf [Gr].

Lemma 3.7 Assume that (3.20) holds. Then

$$
\sup _{e \in(a, b), \epsilon \neq 0}|\epsilon| \mathbb{E}\left(|\mathcal{R}((m, 0),(n, 0) ; e+\mathrm{i} \epsilon)|^{2}\right) \leq C\langle n-m\rangle^{-1-\delta} .
$$

Remark. This result is not stated in [Gr] in the above form. However, it is an immediate consequence of the proof of Lemma 3 in [Gr].

We denote by $\mathcal{A}$ the set of all $C_{0}^{\infty}$-functions with support in $(a, b)$.

Lemma 3.8 Assume that for all $\chi \in \mathcal{A}, R \geq 0$ and $n$,

$$
\lim _{T \rightarrow \infty} \frac{1}{2 T} \int_{-T}^{T} \mathbb{E}\left(\left\|\mathbf{1}_{R} \mathrm{e}^{-\mathrm{i} t H} \chi(H) \delta_{(n, 0)}\right\|^{2}\right) \mathrm{d} t=0 .
$$

Then the property $\mathcal{P}$ holds on $(a, b)$.

Proof. The result follows from the fact that the set $\left\{\delta_{(n, 0)}: n \in \mathbb{Z}\right\}$ is cyclic for $H=$ $H_{0}+V$ for any $V$. 
Lemma 3.9 Assume that $\operatorname{supp} \chi \subset(a, b)$. Then for any $\phi, \psi \in \mathcal{H}$,

$$
\lim _{T \rightarrow \infty} \frac{1}{2 T} \int_{-T}^{T} \mathbb{E}\left(\left|\left(\phi \mid \mathrm{e}^{-\mathrm{i} t H} \chi(H) \psi\right)\right|^{2}\right) \mathrm{d} t=0 .
$$

Proof. By the result of [JL1], the spectrum of $H$ is $P$-a.s. purely a.c. on $(a, b)$, and it follows from the Wiener theorem that

$$
\lim _{T \rightarrow \infty} \frac{1}{2 T} \int_{-T}^{T}\left|\left(\phi \mid \mathrm{e}^{-\mathrm{i} t H} \chi(H) \psi\right)\right|^{2} \mathrm{~d} t=0 \quad P-\text { a.s. }
$$

This relation and the Lebesgue dominated convergence theorem yield the statement.

For any $l \geq 0$, let $\mathbf{1}_{R, l}$ be the characteristic function of the set

$$
\{(n, x):|n| \geq l, 0 \leq x \leq R\} .
$$

We use the same notation for the corresponding multiplication operator.

Lemma 3.10 Assume that for all $\chi \in \mathcal{A}, R \geq 0$ and $n$,

$$
\lim _{l \rightarrow \infty} \limsup _{\epsilon \rightarrow 0}|\epsilon| \int_{\mathbb{R}} \mathbb{E}\left(\left\|\mathbf{1}_{R, l} \mathcal{R}(e+\mathrm{i} \epsilon) \chi(H) \delta_{(n, 0)}\right\|^{2}\right) \mathrm{d} e=0 .
$$

Then the property $\mathcal{P}$ holds on $(a, b)$.

Proof. Since $\mathbf{1}_{R}-\mathbf{1}_{R, l}$ is a finite rank operator, by Lemma 3.9

$$
\lim _{T \rightarrow \infty} \frac{1}{2 T} \int_{-T}^{T} \mathbb{E}\left(\left\|\left(\mathbf{1}_{R}-\mathbf{1}_{R, l}\right) \mathrm{e}^{-\mathrm{i} t H} \chi(H) \delta_{(n, 0)}\right\|^{2}\right) \mathrm{d} t=0,
$$

and so, by Lemma 3.8, if for all $\chi$ with $\operatorname{supp} \chi \subset(a, b), R$ and $n$,

$$
\lim _{l \rightarrow \infty} \limsup _{T \rightarrow \infty} \frac{1}{2 T} \int_{-T}^{T} \mathbb{E}\left(\left\|\mathbf{1}_{R, l} \mathrm{e}^{-\mathrm{i} t H} \chi(H) \delta_{(n, 0)}\right\|^{2}\right) \mathrm{d} t=0,
$$

then $\mathcal{P}$ holds on $(a, b)$. The result now follows from Lemma 2.1.

We are now ready to finish:

Proof of Theorem 3.6. We will prove that relation (3.23) holds for all $\chi \in \mathcal{A}$, all $R$ and all $n$. Let $\chi$ be given and let $[c, d]$ be an interval such that $\operatorname{supp} \chi \subset[c, d] \subset(a, b)$. Since for $e \notin[c, d]$,

$$
\sup _{\epsilon \neq 0} \mathbb{E}\left(\left\|\mathbf{1}_{R, l} \mathcal{R}(e+\mathrm{i} \epsilon) \chi(H) \delta_{(n, 0)}\right\|^{2}\right) \leq C(\operatorname{dist}(e, \operatorname{supp} \chi))^{-2},
$$


to prove (3.23) it suffices to show that

$$
\lim _{l \rightarrow \infty} \limsup _{\epsilon \rightarrow 0}|\epsilon| \sup _{e \in(a, b)} \mathbb{E}\left(\left\|\mathbf{1}_{R, l} \mathcal{R}(e+\mathrm{i} \epsilon) \chi(H) \delta_{(n, 0)}\right\|^{2}\right)=0 .
$$

Let $\tilde{\chi}$ be an almost analytic extension of $\chi$. (For the basic facts about almost analytic extensions we refer the reader to [Da].) By the Helffer-Sjöstrand formula,

$$
\chi(H)=\frac{1}{\pi} \int_{\mathbb{C}} \frac{\partial \tilde{\chi}(z)}{\partial \bar{z}} \mathcal{R}(z) \mathrm{d} x \mathrm{~d} y .
$$

It follows that for any $w \in \mathbb{C}$,

$$
\mathbf{1}_{R, l} \mathcal{R}(w) \chi(H)=\frac{1}{\pi} \int_{\mathbb{C}} A(w, z) \mathrm{d} x \mathrm{~d} y,
$$

where

$$
A(w, z)=\frac{\partial \tilde{\chi}(z)}{\partial \bar{z}} \frac{1}{z-w} \mathbf{1}_{R, l}(\mathcal{R}(w)-\mathcal{R}(z)) .
$$

We will make use of the following properties of $\tilde{\chi}$ (see [Da]):

(a) $\operatorname{supp} \tilde{\chi}$ is a compact set.

(b) $\sup _{z \in \operatorname{supp} \tilde{\chi}}\left|\frac{\partial \tilde{\chi}(z)}{\partial \bar{z}}\right|=O(|\operatorname{Im} z|)$.

Setting $w=e+\mathrm{i} \epsilon$ we derive from (3.25) that

$$
\left\|\mathbf{1}_{R, l} \mathcal{R}(w) \chi(H) \delta_{(n, 0)}\right\| \leq\left\|\mathbf{1}_{R, l} \mathcal{R}(w) \delta_{(n, 0)}\right\| D_{1}(w)+D_{2}(w),
$$

where

$$
D_{1}(w)=\frac{1}{\pi} \int_{\mathbb{C}}\left|\frac{\partial \tilde{\chi}(z)}{\partial \bar{z}}\right| \frac{1}{|w-z|} \mathrm{d} x \mathrm{~d} y
$$

and

$$
D_{2}(w)=\frac{1}{\pi} \int_{\mathbb{C}}\left|\frac{\partial \tilde{\chi}(z)}{\partial \bar{z}}\right| \frac{\left\|\mathcal{R}(z) \delta_{(n, 0)}\right\|}{|w-z|} \mathrm{d} x \mathrm{~d} y .
$$

Now (a) and (b) yield that

$$
\sup _{w \in \mathbb{C}} D_{1}(w)<\infty, \quad \sup _{w \in \mathbb{C}} D_{2}(w)<\infty,
$$


and so

$$
\sup _{e \in(a, b)} \mathbb{E}\left(\left\|\mathbf{1}_{R, l} \mathcal{R}(e+\mathrm{i} \epsilon) \chi(H) \delta_{(n, 0)}\right\|^{2}\right) \leq C \sup _{e \in(a, b)} \mathbb{E}\left(\left\|\mathbf{1}_{R, l} \mathcal{R}(e+\mathrm{i} \epsilon) \delta_{(n, 0)}\right\|^{2}\right)+O(1) .
$$

Let $q=\min (3 s / 2,1+\delta)>1$. Using Lemma 3.4, (3.21), and (3.26), we conclude that

$$
\begin{aligned}
\limsup _{\epsilon \rightarrow 0}|\epsilon| \sup _{e \in(a, b)} \mathbb{E}\left(\left\|\mathbf{1}_{R, l} \mathcal{R}(e+\mathrm{i} \epsilon) \chi(H) \delta_{(n, 0)}\right\|^{2}\right) & \leq C \sum_{|m|>l}\langle n-m\rangle^{-q} \\
& =O\left(l^{1-q}\right)
\end{aligned}
$$

and (3.24) follows.

\subsection{Localization estimate in the strong coupling regime}

In this and the next section we use the Aizenman-Molchanov technique to show that the key estimate (3.21) holds if the disorder is either large or small enough (we follow an elegant presentation of Aizenman-Molchanov theory in [S], see also [JM2]). Throughout this section we assume that the assumptions of Theorem 1.4 hold.

Theorem 3.11 For any $2 / 3<s<1$ there is a constant $\Lambda_{s}>0$ such that for $|\lambda|>\Lambda_{s}$ and $\forall m, n \in \mathbb{Z}$,

$$
\sup _{z \in \mathbb{C}_{ \pm}} \mathbb{E}\left(|\mathcal{R}((m, 0),(n, 0) ; z)|^{s}\right) \leq C\langle n-m\rangle^{-3 s / 2}
$$

Theorem 1.4 is an immediate consequence of Theorems 3.6 and 3.11.

The rest of this section is devoted to the proof of Theorem 3.11. To simplify the notation, we assume that $m=0$ and adopt the shorthand

$$
\mathcal{R}(n ; z):=\mathcal{R}((0,0),(n, 0) ; z) .
$$

A similar argument applies to the other values of $m$. In what follows we fix $s \in(2 / 3,1)$. It follows from Lemma 3.2 that the function $\mathcal{R}(n ; z)$ satisfies the equation

$$
\sum_{k} j(n-k, z) \mathcal{R}(k ; z)+\left(U_{\text {per }}(n)+\lambda V(n)-z\right) \mathcal{R}(n ; z)=\delta_{0 n} .
$$

Then

$$
\mathbb{E}\left(\left|U_{\text {per }}(n)+\lambda V(n)-z\right|^{s}|\mathcal{R}(n ; z)|^{s}\right) \leq \delta_{0 n}+\sum_{k}|j(n-k, z)|^{s} \mathbb{E}\left(|\mathcal{R}(k ; z)|^{s}\right) .
$$


The decoupling lemma of Aizenman-Molchanov (see [AM, AG, Gr, M, JM2]) yields that there is a constant $k_{s}$,

$$
k_{s} \geq 2^{-(1+s)}(1-s)\|p\|_{\infty}^{-s}
$$

such that

$$
k_{s}|\lambda|^{s} \mathbb{E}\left(|\mathcal{R}(n ; z)|^{s}\right) \leq \mathbb{E}\left(\left|U_{\text {per }}(n)+\lambda V(n)-z\right|^{s}|\mathcal{R}(n ; z)|^{s}\right)
$$

Let

$$
g(n, z):=\mathbb{E}\left(|\mathcal{R}(n, z)|^{s}\right),
$$

and let $g(z)$ be the sequence $\{g(n, z)\}$. Note that $g(z) \in l^{\infty}(\mathbb{Z})\left(|g(n, z)| \leq|\operatorname{Im} z|^{-s}\right)$. Relations (3.28) and (3.29) yield that

$$
\left(1-k_{s}^{-1}|\lambda|^{-s} T(z)\right) g(z) \leq k_{s}^{-1}|\lambda|^{-s} \delta_{0}
$$

where $T(z)$ is the operator of convolution by $|j(n, z)|^{s}$. It follows from Lemma 3.1 and (3.18) that

$$
\begin{aligned}
C_{1} & :=\sup _{z \in \mathbb{C}_{ \pm}}\|T(z)\|_{\infty} \\
& =\sup _{z \in \mathbb{C}_{ \pm}} \sum_{n}|j(n, z)|^{s}<\infty
\end{aligned}
$$

where $\|\cdot\|_{\infty}$ is the $l^{\infty}$ operator norm. Thus, if

$$
k_{s}|\lambda|^{s}>C_{1}
$$

then the operator $1-k_{s}^{-1}|\lambda|^{-s} T(z)$ is invertible,

$$
\left(1-k_{s}^{-1}|\lambda|^{-s} T(z)\right)^{-1}=\sum_{m=0}^{\infty}\left(k_{s}^{-1}|\lambda|^{-s}\right)^{m} T(z)^{m},
$$

and $\left(1-k_{s}^{-1}|\lambda|^{-s} T(z)\right)^{-1}$ is positivity preserving (since $T(z)$ is). These observations and relation (3.30) yield that

$$
g(z) \leq k_{s}^{-1}|\lambda|^{-s}\left(1-k_{s}^{-1}|\lambda|^{-s} T(z)\right)^{-1} \delta_{0}
$$

and

$$
g(n, z) \leq k_{s}^{-1}|\lambda|^{-s} \sum_{m=0}^{\infty}\left(k_{s}^{-1}|\lambda|^{-s}\right)^{m}\left(\delta_{0} \mid T(z)^{m} \delta_{n}\right)
$$


By Lemma 3.1 and (3.18), there is a constant $C_{2}$ such that

$$
\sup _{z \in \mathbb{C}_{ \pm}}|j(n, z)|^{s} \leq C_{2}\langle n\rangle^{-3 s / 2}
$$

Now let $C_{3}$ be the constant which appears in Lemma 3.5 for $1+\delta=3 s / 2$. If in addition to $(3.31)$ we have that

$$
k_{s}|\lambda|^{s}>C_{2} C_{3}
$$

then (3.32) and Lemma 3.5 show that for some $C$ and all $n$,

$$
\sup _{z \in \mathbb{C}_{ \pm}} g(n, z) \leq C\langle n\rangle^{-3 s / 2}
$$

This yields Theorem 3.11 with

$$
\Lambda_{s}=\left(\max \left(C_{1}, C_{2} C_{3}\right) / k_{s}\right)^{1 / s} .
$$

\subsection{Localization estimate in the weak coupling regime}

Throughout this section we assume that the assumptions of Theorem 1.5 hold. Let $\alpha>$ $5 / 3$ be such that $\langle x\rangle^{\alpha} p(x) \in L^{\infty}(\mathbb{R})$.

Theorem 3.12 For any $s \in(2 / 3, \min (1, \alpha-1))$ there is a constant $\Lambda_{s}>0$ such that for $|\lambda|<\Lambda_{s}$ and $\forall m, n \in \mathbb{Z}$,

$$
\sup _{z \in \mathbb{C}_{ \pm}} \mathbb{E}\left(|\mathcal{R}((m, 0),(n, 0) ; z)|^{s}\right) \leq C\langle n-m\rangle^{-3 s / 2}
$$

Remark. The restriction on $s$ in terms of $\alpha$ is a technical condition which we need to ensure that the constant $K_{s}$ in the Aizenman-Molchanov decoupling lemma (relation (3.34) below) is finite. For some other conditions which also ensure that $K_{s}<\infty$ see [A, AM, JM2].

Theorem 1.5 follows from Theorems 3.6 and 3.12.

The rest of this section is devoted to the proof of Theorem 3.12. We again assume that $m=0$, adopt the shorthand (3.27), and fix $s \in(2 / 3, \min (1, \alpha-1))$. The resolvent identity and Lemma 3.2 yield that

$$
\mathcal{R}(n, z)=j(n, z)-\sum_{k} j(n-k, z) V(k) \mathcal{R}(k, z)
$$


Thus,

$$
\mathbf{E}\left(|\mathcal{R}(n, z)|^{s}\right) \leq|j(n, z)|^{s}+\sum_{k}|j(n-k, z)|^{s} \mathbf{E}\left(|V(k)|^{s}|\mathcal{R}(k, z)|^{s}\right) .
$$

The decoupling lemma of Aizenman-Molchanov yields that there is a finite constant $K_{s}$ such that for $z \in \mathbb{C}_{ \pm}$

$$
\mathbf{E}\left(|V(k)|^{s}|\mathcal{R}(k, z)|^{s}\right) \leq|\lambda|^{s} K_{s} \mathbf{E}\left(|\mathcal{R}(k, z)|^{s}\right)
$$

Let $g(n, z), g(z), T(z), C_{1}, C_{2}$ and $C_{3}$ be as in the previous section, and let $j_{s}(z)$ be the sequence $\left\{|j(n, z)|^{s}\right\}$. Note that $j_{s}(z)=T(z) \delta_{0}$. Relations (3.33) and (3.34) yield that

$$
\left(1-K_{s}|\lambda|^{s} T(z)\right) g(z) \leq j_{s}(z)=T(z) \delta_{0}
$$

Thus, if

$$
K_{s}|\lambda|^{s}<C_{1}^{-1}
$$

then the operator $1-K_{s}|\lambda|^{s} T(z)$ is invertible on the Banach space $l^{\infty}(\mathbb{Z})$ and its inverse is positivity preserving. Hence,

$$
\begin{aligned}
g(z) & \leq\left(1-K_{s}|\lambda|^{s} T(z)\right)^{-1} T(z) \delta_{0} \\
& \leq K_{s}^{-1}|\lambda|^{-s}\left(1-K_{s}|\lambda|^{s} T(z)\right)^{-1} \delta_{0},
\end{aligned}
$$

and

$$
g(n, z) \leq K_{s}^{-1}|\lambda|^{-s} \sum_{m=0}^{\infty}\left(K_{s}|\lambda|^{s}\right)^{m}\left(\delta_{0} \mid T(z)^{m} \delta_{n}\right)
$$

As in the previous section, if in addition to (3.35) we have that

$$
K_{s}|\lambda|^{s}<\left(C_{2} C_{3}\right)^{-1},
$$

then for some $C$ and all $n$

$$
\sup _{z \in \mathbb{C}_{ \pm}} g(n, z) \leq C\langle n\rangle^{-3 s / 2} .
$$

This yields Theorem 3.12 with

$$
\Lambda_{s}=\left(\min \left(C_{1}^{-1},\left(C_{2} C_{3}\right)^{-1}\right) / K_{s}\right)^{1 / s}
$$




\section{References}

[A] Aizenman, M.: Localization at weak disorder: some elementary bounds. Rev. Math. Phys. 6, 1163 (1994)

[AM] Aizenman, M., Molchanov, S.: Localization at large disorder and at extreme energies: an elementary derivation. Commun. Math. Phys. 157, 245 (1993)

[AG] Aizenman, M., Graf, G.-M.: Localization bounds for an electron gas. J. Phys. A 31, $6783(1998)$

[CS] Chahrour, A., Sahbani, J.: On the spectral and scattering theory of the Schrödinger operator with surface potential. Rev. Math. Phys., 12, 561 (2000)

[CFKS] Cycon, H., Froese, R., Kirsch, W., Simon, B.: Schrödinger Operators. BerlinHeidelberg: Springer-Verlag 1987

[Gr] Graf, G.-M.: Anderson localization and the space-time characteristic of continuum states. J. Stat. Phys. 75, 337 (1994)

[Da] Davies, E.B.: Spectral Theory and Differential Operators. Cambridge: Cambridge University Press 1995

[DS] Davies, E.B., Simon, B.: Scattering theory for systems with different spatial asymptotics on the left and right. Commun. Math. Phys. 63, 277 (1978)

[JL1] Jakšić, V., Last, Y.: Corrugated surfaces and a.c. spectrum, to appear in Rev. Math. Phys.

[JL2] Jakšić, V., Last, Y.: Spectral structure of Anderson type Hamiltonians. Invent. Math. 141, 561 (2000)

[JM1] Jakšić, V., Molchanov, S.: On the surface spectrum in dimension two. Helv. Phys. Acta 71, 629 (1999)

[JM2] Jakšić, V., Molchanov, S.: Localization of surface spectra. Commun. Math. Phys. 208, 153 (1999)

[JM3] Jakšić, V., Molchanov, S.: Wave operators for the surface Maryland model. J. Math. Phys. 41, 4452 (2000)

[JM4] Jakšić, V., Molchanov, S.: On the spectrum of the surface Maryland model. Lett. Math. Phys. 45, 185 (1998) 
[JMP1] Jakšić, V., Molchanov, S., Pastur, L.: On the propagation properties of surface waves. Wave Propagation in Complex Media, IMA Vol. Math. Appl. 96, 143 (1998)

[JMP2] Jakšić, V., Molchanov, S., Pastur, L.: in preparation

[KP] Khoruzenko, B.A., Pastur, L.: The localization of surface states: an exactly solvable model. Physics Reports 288, 109 (1997)

[M] Molchanov, S.: Lectures on Random Media. In Lectures on Probability, ed. P. Bernard, Lecture Notes in Mathematics, 1581, Heidelberg: Springer-Verlag 1994

[MV] Molchanov, S., Vainberg, B.: unpublished

[P] Pastur, L.: Surface waves: propagation and localization. Journées "Équations aux dérivées partielles" (Saint-Jean-de Monts, 1995), Exp. No. VI, École Polytech. Palaisseau (1995)

[RS] Reed, M., Simon, B.: Methods of Modern Mathematical Physics, IV. Analysis of Operators, London: Academic Press (1978)

[S] Simon, B.: Spectral analysis of rank one perturbations and applications. CRM Proc. Lecture Notes, Providence, RI: 8, 1995 